\title{
AN-Encoding Compiler: Building Safety-Critical Systems with Commodity Hardware
}

\author{
Christof Fetzer, Ute Schiffel, and Martin Süßkraut \\ Technische Universtät Dresden \\ Department of Computer Science \\ http://wwwse.inf.tu-dresden.de \\ Dresden, Germany \\ $\{$ firstname.lastname\}@se.inf.tu-dresden.de
}

\begin{abstract}
In the future, we expect commodity hardware to be used in safety-critical applications. However, in the future commodity hardware is expected to become less reliable and more susceptible to soft errors because of decreasing feature size and reduced power supply. Thus, software-implemented approaches to deal with unreliable hardware will be needed. To simplify the handling of value failures, we provide failure virtualization in the sense that we transform arbitrary value failures caused by erroneous execution into fail-stop failures. The latter ones are easier to handle. Therefore, we use the arithmetic AN-code because it provides very good error detection capabilities. Arithmetic codes are suitable for the protection of commodity hardware because guarantees can be provided independent of the executing hardware. This paper presents the encoding compiler EC-AN which applies AN-encoding to arbitrary programs. According to our knowledge, this is the first in software implemented complete AN-encoding. Former encoding compilers either encode only small parts of applications or trade-off safety to enable complete AN-encoding.
\end{abstract}

\section{Introduction}

Historically, hardware reliability has been increasing with every new generation. However, one expects that in the future, decreasing feature size of hardware will not lead to more reliable but to less reliable hardware. Borkar in [8] impressively describes the effects of reduced feature sizes. Even today's CPUs already have a variation in operating frequency of about $30 \%$ which is dealt with by using die binning. But this variability will increase further with decreasing feature sizes. Indeed, [16] shows that even today's large computing systems - e. g., the Los Alamos Neutron Science Center - experience failures because of soft errors. The conclusion is that the uncontrollable variety of the production process will make processor designs more and more unpredictable. Furthermore, smaller transistors age faster and thus become faster unreliable and smaller features are more susceptible to soft errors since supply voltages decrease with decreasing feature 
size. It is expected that the amount of failures caused by soft errors will increase exponentially with every new technology generation.

Nowadays, safety related systems are typically built using special purpose hardware. However, these solutions are expensive because the effort put into design and production is much higher and the number of units is much smaller than for commodity systems [3]. Also, such hardware is usually an order of magnitude slower than commodity hardware because it lags behind new developments. We expect that in the future there will be economic pressure to use commodity hardware for dependable computing. Furthermore, systems will become mixed mode, i. e., both critical and non-critical applications will be executed on the same computer system built from unreliable hardware because it is faster and cheaper. Such mixed mode systems will require new dependability mechanisms which make it possible to cope with the restrictive failure detection capabilities of commodity hardware. One crucial step in providing such mechanisms is failure virtualization, i. e., the transformation of a (more difficult to handle) failure model into another (easier to handle) failure model. We aim at turning difficult to handle erroneous output into easier to handle crash failures.

Encoding software using arithmetic codes facilitates software-implemented hardware error detection. In contrast to replication, arithmetic codes enable also the detection of permanent errors. Furthermore, the error detection capabilities of arithmetically encoded applications can be determined independent of the used hardware (see Sect. 3).

In this paper we present an encoding compiler (EC-AN) which transforms arbitrary integer applications into their AN-encoded versions (see Sect. 3). In contrast to similar previous approaches such as [20,10,34], we encode the whole application with the same powerful code. This includes memory, logical operations, and handling of external functions whose source code is not available for encoding. We do not yet support encoding applications using floating point instructions. Those applications have to be modified to use a integer-based software implementation of floating point instructions. Section 4 evaluates both the runtime overhead generated by our AN-encoding compiler and the error detection capabilities.

\section{Related Work}

Usually soft-error tolerant hardware uses replication of large hardware parts and voting for error detection and correction $[38,27,4]$. Currently research efforts include more sophisticated approaches than simple replication. [18] reuses testing circuitry for error detection and correction and [17] extends hardware with built-in soft error resilience which is able to detect and correct soft errors and even to predict a soon hardware failure. The hardware design presented in [26] on-the-fly replicates executed instructions. [23] checks consistency of data independent parts of instruction fetch and decoding for repeated traces within an application. For this to be useful, it is required that an application consists to large parts of traces which are repeated often. All those approaches only aim 
at protecting the execution logic. Memories have to be protected by separate means such as error correcting codes. Of course this list of hardware approaches is far from complete. There are several more but all of them have in common that custom hardware is typically very expensive - too expensive for application in mixed-mode systems which execute both safety-critical and non-safety critical applications. Furthermore, most of these approaches are in contrast to our approach not able to detect permanent hardware errors. The intention is that by providing a software-implemented error detection mechanism up-to-date hardware can be also used in safety-critical systems which require certification. The precondition is that the error detection probability of the mechanism is independent of the actually used hardware. This can be provided by using arithmetic codes such as the AN-code.

Control flow checking, which can be implemented in hardware $[15,9,6]$ or software $[2,30,7,29]$, provides means to recognize invalid control flow for the executed program, that is, execution of sequences of instructions which are not permitted for the executed binary. In contrast to AN-encoding control flow checking cannot detect errors which do only influence processed data.

Algorithm based fault tolerance $[13,28]$ and self-checking software [36, 5] use invariants contained in the executed program to check the validity of the generated results. This requires that appropriate invariants exist. These invariants have to be designed to provide a good failure detection capability and are not easy - if not impossible - to find for most applications.

Other software approaches work with replicated execution and comparison of the obtained results. The protected software is modified during or before compilation - rarely, dynamic binary instrumentation is used [24]. Replication can be implemented at different levels of abstraction. Some approaches duplicate single instructions and execute them in one thread $[22,19,10,25,24,6]$. Other approaches execute duplicates of the whole program within several threads and provide synchronization means for them $[31,12,32]$. For all those approaches which are based on redundant execution of the same program instructions, it is not possible to provide guarantees with respect to permanent hardware errors or soft errors which disturb the voting mechanism.

Instead of duplication, or additionally, arithmetic codes can be used to detect errors. In that case, the program and the processed data are modified. ED4I [20], for example, duplicates instructions but the duplicated instructions do not process the original data but a k-multiple of it, which is a so-called AN-code. All results of duplicate instructions have to be k-multiples of the original results. In this way, most hardware errors are recognizable. However, whenever a program contains logical operations, the authors choose a factor $\mathrm{k}$ which is a power of two to make those operations encodable. Thereby they reduce the detection capabilities immensely. The resulting code cannot detect bit flips in the higher order bits of data values. But those bits contain the original functional value. Furthermore, the authors do not discuss overflow problems with AN-codes which we pointed out in [33]. Over- and underflows in arithmetic operations are not conserved when AN-encoded values are used e. g. in an addition. If for example 
the result of an addition overflows, ED4I will detect an error. This is a false positive because the $\mathrm{C}$ standard expects over- and underflows to work correctly for unsigned integers, i. e. to form a ring. For signed integers overflows are also required to be correct since the addition of a negative number in the end results in an overflow in its unsigned representation. [10] did also use an AN-code but only for operations which easily can handle encoded values such as additions and subtractions. They did also ignore the over-/underflow issue. Furthermore, the encoding is only applied to registers and not to memory. In the end that leaves supposedly only small parts of applications which are AN-encoded. As should be expected their fault injection experiments show a non-negligible amount of undetected failures for most of the tested applications.

Forin's Vital Coded Processor (VCP) [11] and our previous work Software Encoded Processing (SEP) [34] use an even more powerful arithmetic code which is an AN-code extended with per-variable specific signatures and timestamps. This code does not only facilitate detection of faulty execution of operations or modification of data. It also detects the usage of wrong operands which might be caused by address line errors and the usage of wrong operators. VCP adds the encoding on source code level. But VCP can only be applied to applications which do not use dynamic memory and make no use of instructions other than arithmetic operations add, sub, and mult without over- or underflows. SEP on the other hand, encodes applications on runtime and can be applied to arbitrary applications. SEP generates very high runtime overhead. Furthermore, support for encoded logical operations, shift operations, casts, unaligned memory access and arbitrary external functions is not included in SEP.

This paper presents the encoding compiler EC-AN which in contrast to [11, $20,10,34]$ encodes arbitrary applications completely with an AN-code. In the future, we will extend this compiler to support AN-code with signatures and timestamps.

\section{AN-Encoding of an Application}

A long known technique to detect hardware errors during runtime are arithmetic codes. Arithmetic codes add redundancy to processed data which results in a larger domain of possible words. The domain of possible words contains the smaller subset of valid code words. Arithmetic codes are conserved by correct arithmetic operations, i. e., a correctly executed operation taking valid code words as input produces a result which is also a valid code word. On the other hand, faulty arithmetic operations destroy the code with a very high probability, i. e., result in a non-valid code word [1].

The $A N$-code is one of the most widely known arithmetic codes. Encoding is done by multiplying the information part $x_{f}$ of variable $\mathrm{x}$ with a constant $A$. Thereby, the encoded version $x_{c}$ is obtained. Only multiples of $A$ are valid code words and every operation processing $\mathrm{AN}$-encoded data has to conserve this property. Code checking is done by computing the modulus with $A$. which is 
zero for a valid code word. A variable is checked before it is used as a parameter of an external function, or before it influences data or control flow.

If $A$ requires $k$ bits and we encode values with a maximum size of $n$ bits, we need $n+k$ bits to store encoded values. Assuming a failure model with equally distributed bit flips and that the Hamming distance between all code words is constant the resulting probability $p$ of not detecting an error is: $p=$ $\frac{\text { number of valid code words- } 1}{\text { number of possible words }} \approx \frac{2^{n}}{2^{n+k}}=2^{-k}$ Thus, the error detection capability is independent of the actually used hardware - it just depends on the choice of $A$. $A$ should be as large as possible and should not be a power of two because then multiplication by $A$ only shifts the bits to the left and no bitflips in the higher bits can be detected. Furthermore, $A$ should have as few factors as possible to reduce the probability of undetected operation errors. Hence, most large prime numbers are a good choice for $A$.

For encoding a program with an AN-code, every instruction and every variable has to be replaced with its appropriate AN-encoded version. We use a 64-bit data type for encoded values and support encoding up to 32-bit integers. That leaves 32-bit for $A$. The encoded value is always a 64-bit type regardless of the bitwidth of the unencoded value. We do the instrumentation statically on compilation time because: (1) The protection starts with the encoding. The earlier the encoding is done, the larger is the sphere of protection. Any errors introduced by the steps following encoding, e. g., lowering the code to an executable binary, are detectable. (2) We do not introduce further slowdowns because of dynamic instrumentation. See [35] for a detailed discussion of advantages and disadvantages of encoding on compile vs on runtime. We implement compile time encoding using the LLVM compiler framework [14]. We encode LLVM's bitcode which is a static single assignment assembler-like language. It clearly distinguishes static data flow which occurs within LLVM-registers and dynamic data flow which is implemented using load and store instructions accessing memory. The advantage of LLVM's bitcode, in comparison to any native assembler, is its manageable amount of instructions for which we have to provide encoded versions and the LLVM framework for analyzing and modifying LLVM bitcode.

For example, the following simple C-code snippet:

if $(\mathrm{d}+\mathrm{e}==\mathrm{b})$ return 1 ; else return 0 ;

is equivalent to the following LLVM bitcode

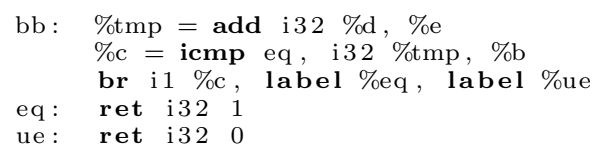

whose encoded version with enlarged data types, replaced operations, and encoded constants looks like that:

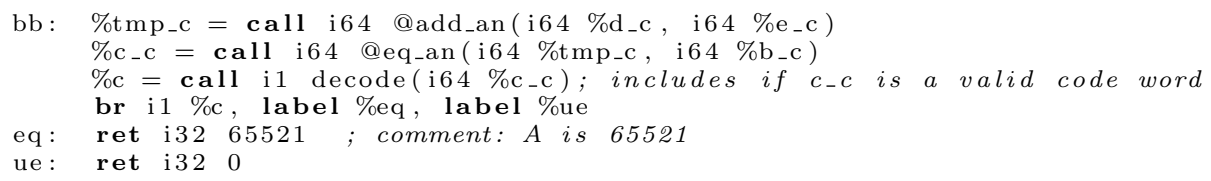


Note, that with AN-encoding the control flow itself, i. e. the actual jump, is not encoded. Only the condition is checked if it is a valid code word. In the future, we will extend the encoding compiler EC-AN with signatures as used by $[11,34]$. Thereby, we will provide control flow checking within basic blocks and between basic blocks. In this case, a variable is not only multiplied with $A$ but additionally a variable-specific signature is added.

For AN-encoding LLVM bitcode, we solved the following problems:

(1) We need encoded versions of all operations supported by LLVM. For arithmetic, logical boolean and shift operations we did reuse our already existing but improved implementations for the AN-code with signatures which were presented in [33]. But for type casting, arithmetic right shift and bitwise logical operations new solutions had to be developed since they were not supported by previous solutions.

(2) For the encoding of memory content, a specific word size had to be chosen: All memory accesses have to be aligned to that word size because only whole encoded words can be read.

(3) We have to provide encoded versions of all constants and intialization values.

(4) We have to handle calls to external libraries. Those are not encoded because we have no access to their sources.

Arithmetic Operations. For arithmetic operations we use by hand encoded operations These operations take encoded operands and produce valid encoded results without decoding the operands for the computation. [33] describes the implementation of these encoded arithmetic operations for an AN code with signatures. The described problems and solutions can be applied to AN codes likewise. Since the operations have to implement the expected overflow behavior of normal integer operations, i. e., modulo arithmetic, their implementation is non-trivial and will generate noticeable slowdowns. Furthermore, the multiplication of two encoded values of 64 bit size results in a 128-bit value. The division requires to multiply the dividend with $A$ before executing the actual division which then has to be 128-bit division. The usage of 128-bit integer operations results in especially large slowdowns for multiplication and division. Our new overhead measurements are presented in Sect. 4.

Replacement Operations. Since encoding by hand is a tedious and errorprone task we automated as much of the remaining encoding tasks as possible. Thus, we provide a library of so-called replacement operations. Those contain implementations of the following operations: shifts, casts, bitwise logical operations, (unaligned) memory accesses and the LLVM instruction getElementPtr which implements address calculation. The replacement operations are written in such a way that they can be automatically encoded by the actual encoding pass of our encoding compiler EC-AN. Before executing the encoding pass, the EC-AN replaces all otherwise non-encodable operations with their appropriate encodable replacement operations which are described in the following.

Shift Operations. Encoded versions of arithmetic and logic shift operations can be implemented using division and multiplication with powers of two since $a<k$ is equivalent to $a * 2^{k}$ and $a \gg k$ is equivalent to $\frac{a}{2^{k}}$. For obtaining $2^{k}$ we 
use a tabulated power-of-two function with precomputed values. An arithmetic right-shift additionally requires a sign-extension to be made if the shifted value is negative. The following pseudo-code represents the encodable variant of the 8-bit arithmetic right shift operations. It shifts val $\mathrm{k}$ bits to the right:

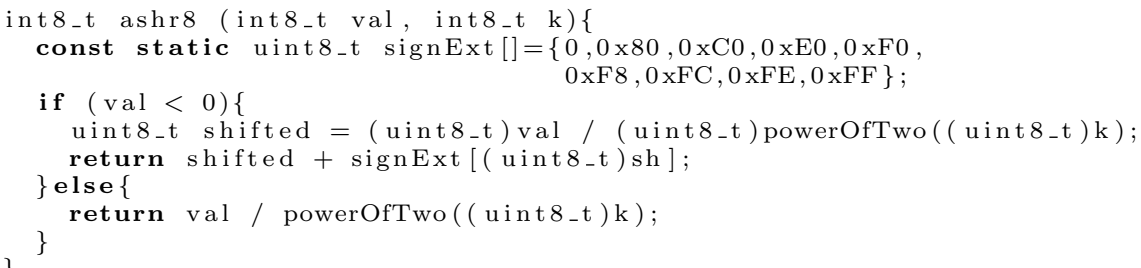

Cast Operations. Cast operations also have to be emulated using encoded operations. For downcasts, i. e., casts from a larger to smaller-sized type, this can be done by doing a modulo computation which can be implemented in an encodable way using division, multiplication, and subtraction. If, for example, the 32-bit integer $a$ is downcasted to 8 bit, we compute its new value using the encoded version of $a \bmod 2^{8}$. Unsigned upcasts from smaller to larger unsigned types require no further actions. Signed upcasts need to check if the casted number is negative and if so, a sign extension has to be made by adding the appropriate sign bits. Assume the 8-bit integer $a$ is negative, i. e., its sign bit is set, and it is casted to a 16-bit signed integer, we would have to execute the encoded version of $a=f f 00_{h e x}+a$.

Logical Operations. Encoding boolean logical operations can be emulated using arithmetic operations. This implementation of boolean logical operations requires that the processed functional values are either 1 (for true) or 0 (for false):

\begin{tabular}{ll|ll} 
original & emulation & original & emulation \\
\hline $\mathrm{x} \mathrm{\|} \mathrm{y}$ & $x+y-x * y$ & $! \mathrm{x}$ & $1-x$ \\
$\mathrm{x} \& \& \mathrm{y}$ & $x * y$ & $\mathrm{x}{ }^{\wedge} \mathrm{y}$ & $(x+y) \bmod 2$
\end{tabular}

Realizing encoded bitwise logical operations is more difficult. The naive approach using shift and addition operations to compute every bit individually would generate a huge overhead. We decided to use tabulated results of logical operations. Since tabulating all possible results for 8-, 16-, and 32-bit integers would require way too much memory, we only tabulate smaller chunks: 16-bit for the not operation and 8-bit for the other operations. To combine those chunks, we use arithmetic operations. The following pseudocode demonstrates this approach for the 32-bit not operation. The other bitwise logical operations use a two dimensional array and smaller chunks but are otherwise implemented similarly:

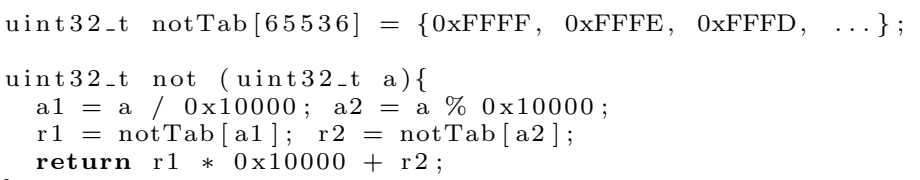


Memory Encoding. We chose to encode the memory at 32-bit granularity because we assume that most programs mainly operate on 32-bit values. This means every 32-bit word in memory is stored as an encoded 64-bit word. Thus, we need to adapt every load and store operation because they have to map the original address to the appropriate address of the encoded value. Furthermore, all memory accesses in the program to encode have to be aligned to 32-bit boundaries. Thus, we replace before encoding the program all unaligned loads and stores with implementations which implement those operations using aligned loads and stores. As Fig. 1 demonstrates, this requires for an unaligned 32-bit load to read both affected 32-bit words (at addresses 64 and 68) and to use logical operations to put together the result 32-bit word (at address 66).
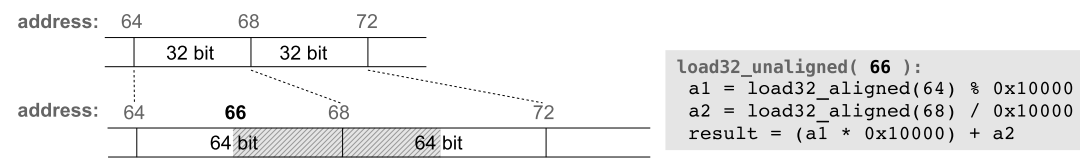

Fig. 1. Execution of an unaligned load at address 66. The upper part represents the memory layout of the original program, the lower part that of the encoded program but with unmapped addresses.

Unaligned stores require to read at least one affected 32-bit word, e. g., when executing an 8-bit store, and in worst case two if it is a 32-bit unaligned store. The read words are than modified accordingly and written back. To prevent accessing unallocated memory when executing an unaligned store, we adapt the size of all allocated memory regions to be a multiple of 32 bits. Note that we zero-initialize all allocated memory regions.

Since pointers are treated like other data items and we restrained the encodable data size to at most 32 bit, we have to ensure that when compiling and executing on 64-bit architectures the encountered addresses do not exceed the 32-bit address range. Therefore, we brought all memory allocations under our control.

getElementPtr. The getElementPtr LLVM instruction implements address calculations. It does not access memory. Its operands are a pointer to a (possibly nested) structure or an array and several indices which describe the indexed element of the structure or array. Before encoding an application, we replace all getElementPtr instructions with explicit address computations. Therefore, we take the architecture dependent type sizes into account and replace getElementPtr using addition and multiplication. This step makes the resulting LLVM binary architecture dependent.

Constant Encoding. LLVM enables us to find and modify all initialization values of variables and constants. We replace them with appropriate multiples of $A$. In LLVM-bitcode non-integer constants are accessed using load and store, i. e. as memory. Thus, those constants are encoded according to our rules for 


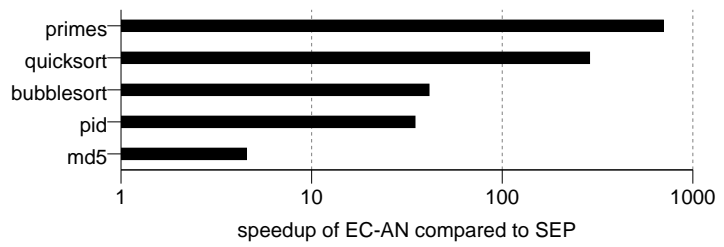

Fig. 2. Speedup of EC-AN (AN-code) compared to SEP (AN-code with signatures and timestamps).

memory, that is they are divided into 32-bit chunks. Therefore, we ensure that their size is a multiple of 32 bit.

External Calls. In contrast to dynamic binary instrumentation, static instrumentation does not allow for protection of external libraries whose source code is not available on compilation time. For calls to those libraries, we currently provide hand-coded decoding wrappers which decode parameters and after executing the unencoded original, encode the obtained results. For implementing those wrappers, we rely on the specifications of the external functions. Using the specifications of external libraries, those wrappers can be generated automatically.

Last, we want to point out that AN-encoding leads to unexpected performance modifications. Some operations whose unencoded versions are very fast, such as, casts, shifts, bitwise logical operations, multiplications and divisions suddenly induce very large overheads. Therefore, programmers should avoid these operations if developing explicitly for a system protected by AN-codes.

\section{Evaluation}

We evaluated our approach using five small examples:

(1) md5 calculates the md5 hash of a 20,000 characters long string,

(2) quicksort sorts an array of 10000 integers,

(3) bubblesort sorts an array of 1000 integers,

(4) primes calculates all prime numbers up to 10,000 and

(5) pid runs 500, 000 steps of a Proportional-Integral-Derivative controller [37]. Performance. Figure 2 compares the runtimes of an on compile time ANencoded application to an on runtime encoded application using SEP by depicting the achieved speedup. In general it can be seen that the compiled version is much faster than the interpreted version. That has several reasons: (1) We are comparing an AN-code with an AN-code with signatures and timestamps. The latter one induces larger overheads for all encoded operations. After extending the encoding compiler EC-AN to an AN-code with signatures and timestamps, the resulting speedups surely will be smaller. (2) With EC-AN all encoding is done at compile time as an LLVM compiler extension. Thus, the overhead at runtime compared to SEP is smaller because the binary is natively executed. 


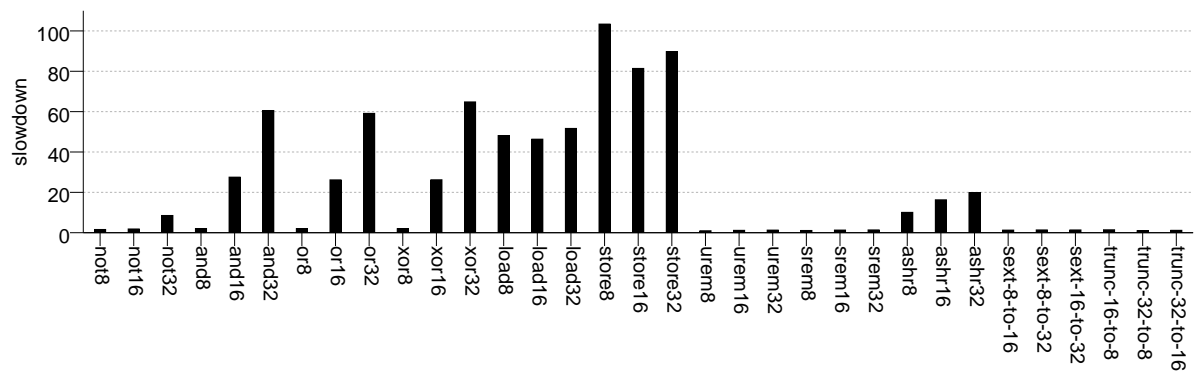

Fig. 3. Slowdowns of encodable versions of replacement operations compared to their native versions.

We observe that the obtained speedups largely depend on the executed program. The reason for this is the incompleteness of SEP which does not support encoded versions of logical operations, shift operations, casts, and unaligned memory access. Those operations are just executed unencoded in SEP while they are encoded with the help of the described replacement operations by EC-AN. Those operations generate already in their encodable but yet unencoded version large slowdowns compared to their native versions. See Fig. 3. Thus, applications using many of those operations such as md5 result in a smaller speedup.

Furthermore, we see that the encodable versions of unaligned loads (load $x$ ) and stores $(\operatorname{store} x)$ are also very expensive. Arithmetic right shifts (ashr $x$ ) are not as expensive but still between 10 and 20 times slower than their native counterparts. Whereas the encodable versions of the signed and unsigned modulo operations ( $\operatorname{srem} x$ and $\operatorname{urem} x$ ), and upcast and downcast operations (sext- $x-$ to $-y$ and trunc- $x-$ to- $y$ ) are very cheap.

Figure 4 evaluates the slowdowns of our AN-encoded arithmetic operations compared to their native counterparts. We compare two versions: One implements the required 128-bit operations in software while the other one uses the SSE-extensions of the processor. Both are as far as possible compiled using LLVM with optimizations.

Since 128-bit operations are only used for multiplications and divisions, we see only for them a difference - but that is immense. Nevertheless, the slowdowns are very large. For the future, we plan to extend EC-AN so that it supports adaptive encoding. As stated in [21] not all calculations are equally important. We want to enable the programmer to identify safety-critical parts of an application. The encoding will then only be applied to those parts thereby reducing the performance impact. The rest of the program (e.g. book-keeping, user interface) could run unencoded with native speed.

Furthermore, those safety-critical parts should be written for encodability: All memory accesses should be aligned which would remove the overhead introduced by implementing encoded aligned memory accesses. Developers should try to avoid bitwise logical operations and shifts. By sticking to the same data type, they can avoid explicit and implicit casts. Depending on the application, 


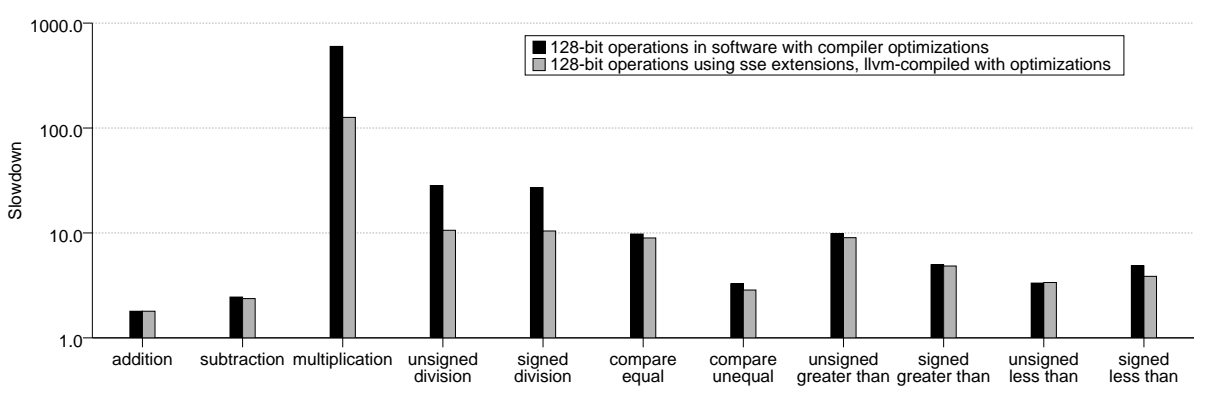

Fig. 4. Slowdowns of encoded arithmetic operations compared to their native versions for two versions: (1) 128-bit arithmetic implemented as software library and (2) implemented using the processors SSE extensions.

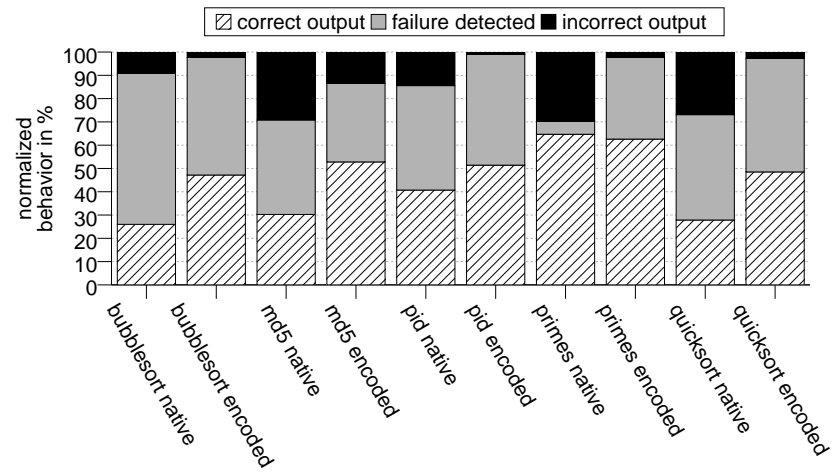

Fig. 5. Error injection results for injection of: operation, operand and lost update errors.

programmers would like to check by themselves for overflows. In this case, they could use faster encoded operations without overflow correction.

Error Detection. Figure 5 shows the results of our error injection experiments. The used error injection tool was also implemented using LLVM. It inserts the following types of errors according to Forin's error model [11]:

(1) operation errors: the result of an operation is wrong.

(2) operand errors: an operand is modified, e. g. by an bitflip.

(3) lost updates: a store operation is unsuccessful.

In each injection run exactly one error is inserted. We performed 3000 injection runs for the native, i. e. unprotected versions, and the AN-encoded versions of our programs.

We see that for all runs a large part of the injections does not result in a failure (correct output), that is the program neither crashed nor produced erroneous results. Failure detected means that the application crashed. That is our intended error model. We want to turn difficult to handle value failures 
(incorrect output) into easier to detect and thus to handle crash failures. For all AN-encoded versions of the programs the amount of runs which produced incorrect output is smaller than for the unprotected versions. But it is not zero for several reasons:

(1) An AN-code does not provide protection from exchanged operand errors, e. g., a store which reads the wrong address, neither from lost updates or modified control flow.

(2) In contrast to SEP external functions such as printf are unprotected.

We also see that the relation of the amount of undetected errors between native and $\mathrm{AN}$-encoded version varies largely with the executed program. For md5 the improvement compared to native is rather small compared to the other programs. We believe, the reason for this is that the AN-code does not detect loads and stores to/from the wrong address.

\section{Conclusion}

We presented the AN-encoding compiler EC-AN which applies the arithmetic AN-code to arbitrary programs. In contrast to earlier approaches the encoding is applied to the whole program. In contrast to existing solutions we do also encode bitwise logical operations completely using a powerful AN-code. Existing solutions did either not encode bitwise logical operations at all $[11,10,34]$ or did if a program contained them switch to a much less safe code variant for the whole program [20]. The measurements show that the approach is successful in detecting errors but there are still undetected incorrect outputs in the presence of errors. This further motivates our ongoing work to extend EC-AN to support AN-encoding with signatures and timestamps. Compared to the in principle safer SEP [34] the runtime overhead produced by with EC-AN encoded programs is far lower. This is promising for EC-AN's extension to support signatures and timestamps which will make its error detection capabilities equal to those of SEP and in the end even better since EC-AN does a more complete encoding. Nevertheless, the observed slowdowns require actions to be taken to mitigate them. We will research the concept of adaptive safety, that is to apply the encoding only to the most safety-critical parts of an application.

\section{References}

1. A. Avizienis. Arithmetic error codes: Cost and effectiveness studies for application in digital system design. In Transactions on Computers, 1971.

2. S. Bagchi, Z. Kalbarczyk, R. Iyer, and Y. Levendel. Design and evaluation of preemptive control signature(PECOS) checking. IEEE Transactions on Computers, 2003.

3. Hugh J. Barnaby. Will radiation-hardening-by-design (RHBD) work? Nuclear and Plasma Sciences, Society News, 2005.

4. David Bernick, Bill Bruckert, Paul Del Vigna, David Garcia, Robert Jardine, Jim Klecka, and Jim Smullen. Nonstop advanced architecture. Proceedings of the International Conference on Dependable Systems and Networks (DSN), 2005. 
5. M. Blum, M. Luby, and R. Rubinfeld. Self-testing/correcting with applications to numerical problems. In STOC '90: Proceedings of the twenty-second annual ACM symposium on Theory of computing, New York, NY, USA, 1990. ACM Press.

6. C. Bolchini, A. Miele, M. Rebaudengo, F. Salice, D. Sciuto, L. Sterpone, and M. Violante. Software and hardware techniques for SEU detection in IP processors. J. Electron. Test., 24(1-3):35-44, 2008.

7. Edson Borin, Cheng Wang, Youfeng Wu, and Guido Araujo. Software-based transparent and comprehensive control-flow error detection. In Proceedings of the International Symposium on Code Generation and Optimization (CGO), pages 333-345, Washington, DC, USA, 2006. IEEE Computer Society.

8. Shekhar Borkar. Designing reliable systems from unreliable components: The challenges of transistor variability and degradation. IEEE Micro, 2005.

9. Mihai Budiu, Úlfar Erlingsson, and Martín Abadi. Architectural support for software-based protection. In ASID '06: Proceedings of the 1st workshop on Architectural and system support for improving software dependability, pages 42-51, New York, NY, USA, 2006. ACM.

10. Jonathan Chang, George A. Reis, and David I. August. Automatic instructionlevel software-only recovery. In Proceedings of the International Conference on Dependable Systems and Networks (DSN), Washington, USA, 2006.

11. P. Forin. Vital coded microprocessor principles and application for various transit systems. In IFA-GCCT, pages 79-84, Sept 1989.

12. Mohamed Gomaa, Chad Scarbrough, T. N. Vijaykumar, and Irith Pomeranz. Transient-fault recovery for chip multiprocessors. International Symposium on Computer Architecture, 2003.

13. Kuang-Hua Huang and Jacob A. Abraham. Algorithm-based fault tolerance for matrix operations. IEEE Trans. Computers, 33(6):518-528, 1984.

14. Chris Lattner and Vikram Adve. LLVM: A compilation framework for lifelong program analysis \& transformation. In Proceedings of the international symposium on Code generation and optimization (CGO), page 75, Washington, DC, USA, 2004. IEEE Computer Society.

15. Xiaobin Li and Jean-Luc Gaudiot. A compiler-assisted on-chip assigned-signature control flow checking. In Asia-Pacific Computer Systems Architecture Conference, 2004.

16. S.E. Michalak, K.W. Harris, N.W. Hengartner, B.E. Takala, and S.A. Wender. Predicting the number of fatal soft errors in Los Alamos National Laboratory's ASC Q supercomputer. In IEEE Transactions on Device and Materials Reliability, 2005 .

17. S. Mitra. Globally optimized robust systems to overcome scaled CMOS reliability challenges. Design, Automation and Test in Europe (DATE '08), 2008.

18. Subhasish Mitra, Norbert Seifert, Ming Zhang, Quan Shi, and Kee Sup Kim. Robust system design with built-in soft-error resilience. Computer, 38(2):43-52, 2005.

19. B. Nicolescu and Raoul Velazco. Detecting soft errors by a purely software approach: Method, tools and experimental results. In Design, Automation and Test in Europe (DATE '03), 2003.

20. Nahmsuk Oh, Subhasish Mitra, and Edward J. McCluskey. ED4I: Error detection by diverse data and duplicated instructions. IEEE Trans. Comput., 51, 2002.

21. Karthik Pattabiraman, Vinod Grover, and Benjamin G. Zorn. Samurai: protecting critical data in unsafe languages. In Eurosys '08: Proceedings of the 3rd ACM SIGOPS/EuroSys European Conference on Computer Systems 2008, pages 219232, New York, NY, USA, 2008. ACM. 
22. Maurizio Rebaudengo, Matteo Sonza Reorda, Massimo Violante, and Marco Torchiano. A source-to-source compiler for generating dependable software. Proceedings of the First IEEE International Workshop on Source Code Analysis and Manipulation (SCAM), 2001.

23. Vimal Reddy and Eric Rotenberg. Inherent time redundancy (itr): Using program repetition for low-overhead fault tolerance. In DSN '07: Proceedings of the 37th Annual IEEE/IFIP International Conference on Dependable Systems and Networks, Washington, DC, USA, 2007. IEEE Computer Society.

24. George A. Reis, Jonathan Chang, David I. August, Robert Cohn, and Shubhendu S. Mukherjee. Configurable transient fault detection via dynamic binary translation. In Proceedings of the 2nd Workshop on Architectural Reliability (WAR), 2006.

25. George A. Reis, Jonathan Chang, Neil Vachharajani, Ram Rangan, David I. August, and Shubhendu S. Mukherjee. Design and evaluation of hybrid fault-detection systems. In ISCA '05: Proceedings of the 32nd annual international symposium on Computer Architecture, Washington, USA, 2005. IEEE Computer Society.

26. Eduardo Luis Rhod, Carlos Arthur Lisbôa, Luigi Carro, Matteo Sonza Reorda, and Massimo Violante. Hardware and software transparency in the protection of programs against SEUs and SETs. J. Electron. Test., 24(1-3):45-56, 2008.

27. T.J. Slegel, R.M. Averill, M.A. Check, B.C. Giamei, B.W. Krumm, W.H. Krygowski, C.A.and Li, J.S. Liptay, J.D. MacDougall, T.J. McPherson, J.A. Navarro, E.M. Schwarz, K. Shum, and C.F. Webb. IBM's S/390 G5 microprocessor design. IEEE Micro, 19:12 - 23, 1999.

28. V.K. Stefanidis and K.G. Margaritis. Algorithm based fault tolerance : Review and experimental study. In International Conference of Numerical Analysis and Applied Mathematics, 2004.

29. Ramtilak Vemu and Jacob A. Abraham. CEDA: Control-flow error detection through assertions. In IOLTS '06: Proceedings of the 12th IEEE International Symposium on On-Line Testing, Washington, DC, USA, 2006. IEEE Computer Society.

30. Rajesh Venkatasubramanian, John P. Hayes, and Brian T. Murray. Low-cost online fault detection using control flow assertions. Proceedings of the 9th IEEE On-Line Testing Symposium (IOLTS), 00:137, 2003.

31. T. N. Vijaykumar, Irith Pomeranz, and Karl Cheng. Transient-fault recovery using simultaneous multithreading. SIGARCH Comput. Archit. News, 30(2):87-98, 2002.

32. Cheng Wang, Ho seop Kim, Youfeng Wu, and Victor Ying. Compiler-managed software-based redundant multi-threading for transient fault detection. In International Symposium on Code Generation and Optimization (CGO), 2007.

33. Ute Wappler and Christof Fetzer. Hardware failure virtualization via software encoded processing. In 5th IEEE International Conference on Industrial Informatics (INDIN 2007), 2007.

34. Ute Wappler and Christof Fetzer. Software encoded processing: Building dependable systems with commodity hardware. In The 26th International Conference on Computer Safety, Reliability and Security (SafeComp 2007), 2007.

35. Ute Wappler and Martin Müller. Software protection mechanisms for dependable systems. Design, Automation and Test in Europe (DATE '08), 2008.

36. Hal Wasserman and Manuel Blum. Software reliability via run-time resultchecking. J. ACM, 1997.

37. Tim Wescott. PID without a PhD. Embedded Systems Programming, 13(11), 2000.

38. Y Yeh. Triple-triple redundant 777 primary flight computer. In In Proceedings of the 1996 IEEE Aerospace Applications Conference 1, pages 293-307, 1996. 